\title{
Ki-67 proliferative index correlation to the immunohistochemistry profile in early female breast cancer: a review of 515 cases
}

\section{Corresponding author:}

Abdalla Saad Abdalla Al-Zawi, Basildon \& Thurrock University Hospital, Nethermayne, SS16 5NL Basildon, ESSEX, United Kingdom, e-mail: abdalasaad@gmail.com
Medical Research Journal 2021; Volume 6. Number 2, 108-113 DOI: 10.5603 MRJ.2021.0026 Copyright $\odot 2021$ Via Medica ISSN 2451-2591 e-ISSN 2451-4101

\begin{abstract}
Introduction: Many biological markers are used as prognostic and predictive indicators in invasive breast cancers management. Among them, tumour size, grade, patho-morphological subtype, hormone receptors status and HER2 receptor expression in addition to Ki-67 proliferative index. Also, they play a key role in adjuvant treatment decision making. Our aim was to evaluate the association between Ki-67 proliferative index and breast cancer immunological subtype.

Material and methods: A total of 515 early invasive patients were enrolled, tumour biological characteristics as histopathological subtype, immune-histo-chemistry (ER,PR,HER2) status and Ki-67 proliferation index values have been collected. The Ki-67 index level of $20 \%$, was used as the cut-off point to differentiate between low and high Ki-67 expression levels. Statistical analysis has been performed using the Chi square test online tool.

Results: In this cohort, about $42 \%, 33 \%, 7 \%$, and $18 \%$ of the cases were grouped as luminal A-like, luminal B-like, HER2 enriched subtype, and triple-negative, respectively. All luminal A-like patients had Ki-67 level less than $20 \%$. About $3 \%$ of the cohort, are luminal B-like tumours with $\mathrm{Ki}-67$ level less than $20 \%$, where $30.3 \%$ of the patients were luminal B-like tumours with Ki-67 level $\geq 20 \%$. In HER2 enriched subtype, Ki67 of $<20 \%$ level seen in $1.9 \%$ of cases, and Ki-67 levels $\geq 20 \%$ was observed in $5.2 \%$ of the cases. In the triple-negative group, Ki-67 was $20 \%$ or higher in $16 \%$ of cases, and only $1.7 \%$ of patients had Ki-67 level less than $20 \%$.

Conclusion: Luminal A-like tumours were the most frequently encountered subtype, they have low Ki-67 levels and are known to be of a low histological grade tumour, and usually associated with a good prognosis. Also, data indicates that high Ki-67 levels are seen more often in Luminal B-Like breast cancers as well as in triple-negative breast cancers and HER2 enriched tumours.
\end{abstract}

Key words: breast neoplasms, Ki-67 proliferation index, eestrogen receptor, Progesterone receptor, HER2 Med Res J 2021; 6 (2): 108-113

\section{Introduction}

According to $2018 \mathrm{WHO}$ reports, breast malignancy is the most common cancer among the female gender with the lifetime risk of $12 \%$. It is followed by colorectal, lung, cervical and thyroid cancer as the most common cancers in women [1,2]. The previous reports showed that breast cancer immuno-histochemical subtypes have a real impact on the disease prognosis and to the response to hormonal blockade treatment and chemotherapy. In 1983, Gerdes et al., described the $\mathrm{Ki}-67$ protein, which is a nonhistone protein, works as a surfactant and helps the chromosomes to preserve discrete confirmations in a condensed state. If the Ki67 coating layer is missing, during the metaphase of the cell cycle, the chromosomes remodelled into an unshaped or amorphous mass, where the cell division and replication will be compromised [3]. Ki-67 proliferation index significance has been widely studied, it is used as a diagnostic, prognostic and predictive marker in the 
management of cancers originated in breast, pancreas, colon and prostate in addition to endometriosis [4-8]. Tumours that express a higher levels of Ki-67 proliferation index have a worse prognosis than tumors that express lower levels [9].

\section{Material and methods}

A total of 515 patients were included in this study. All the patients had an early/loco-regional breast cancer diagnosis in the period between 2014-2020. Informed consent from the patients was not necessary, however, the study results were a secondary outcome of a regional audit which was approved by the Institutional Review Board under the number GSART336. After the diagnosis, they underwent the recommended management. Patients with metastatic disease at presentation or not fit for curative management has been excluded. The medical records have been retrieved, the data related to the patients' demographics, tumour biological characteristics as histopathological subtype, immune-histo-chemistry (ER, PR, HER2) status and Ki-67 proliferation index values have been collected and analysed. The Ki-67 index level of $20 \%$, was used as the cut-off point to differentiate between low and high Ki-67 expression. The statistical analysis has been performed using Chi-square test on excel, the calculated p-values of less than 0.05 were considered statistically significant.

\section{Results}

Our retrospective study was carried out on 515 patients with operable early invasive breast carcinomas. The age span of the cohort, ranges between 27-97 years, with a mean of 63 years. The different clinico-histo-pathological parameters of breast cancer varieties are shown in (Tab. 1). Among the 515 cases, 297 patients aged less than 70 years (57.6\%) and $218(42.3 \%)$ aged more than 70 years. In terms of the histopathological features, 436 cases (84.6\%) were IDC, NST (invasive ductal carcinoma, of no special type), 48 tumours showed invasive lobular carcinoma (ILC) (9.3\%), 12 (2.3\%) cases were papillary carcinoma and $9(1.7 \%)$ patients had mucinous carcinoma. About $49 \%$ (254) of the cases were grade II, 32\% (166) grade III and about $18.4 \%$ (95) were grade I tumours (Tab. 1). With regard to the female sex hormone expression, the ER (Oestrogen receptor) positive expression tumours were $72.6 \%$ and PR (progesterone receptors) positive expression detected in $58 \%$ of the cases. Approximately $17 \%$ of tumours were HER2+ (score $3+$ or amplified FISH test), where $18 \%$ of cases were triple-negative phenotype. We have used $20 \%$ as the cutting point for Ki-67 proliferation expression. Ki-67 nuclear positivity of $\geq 20 \%$ was detected in $52 \%$ (262) of the cases (Tab. 1). We have classified the 515 cases according to the available immune-phenotyping results into four groups: 1) luminal-A like (ER-positive or PR-positive and HER2-negative), 2) luminal-B like (ER-positive and/or

Table 1. Tumour characteristics and its relation to different immuno-histochemical subtypes, \% (n)

\begin{tabular}{|c|c|c|c|c|c|c|}
\hline Criteria & All & Luminal A-Like & Luminal B-like & HER2 Enriched & Triple-negative & $P$ value \\
\hline \multicolumn{7}{|l|}{ Age (years) } \\
\hline$<70$ & $57.6 \%(297)$ & $23 \%(117)$ & $18 \%(93)$ & $4.85 \%(25)$ & $12 \%(62)$ & \multirow[b]{2}{*}{$0^{*}$} \\
\hline$\geq 70$ & $42.3 \%(218)$ & $19.2 \%(99)$ & $15.1 \%(78)$ & $2.3 \%(12)$ & $5.6 \%(29)$ & \\
\hline \multicolumn{7}{|c|}{ Tumour grade } \\
\hline G I & $18.4 \%(95)$ & $15 \%(78)$ & $02.9 \%(15)$ & 0 & $0.38 \%(2)$ & \multirow{3}{*}{$<0.00001$} \\
\hline$G \|$ & $49 \%(254)$ & $25 \%(128)$ & $18 \%(95)$ & $02.3 \%(12)$ & $03.7 \%(19)$ & \\
\hline G III & $32 \%(166)$ & $2 \%(10)$ & $12 \%(61)$ & $4.85 \%(25)$ & $13.4 \%(70)$ & \\
\hline \multicolumn{7}{|l|}{ Ki-67 } \\
\hline$<20 \%$ & $48 \%(249)$ & $42 \%(216)$ & $2.9 \%(15)$ & $1.9 \%(10)$ & $1.7 \%(9)$ & \multirow[b]{2}{*}{0.0003} \\
\hline$\geq 20 \%$ & $52 \%(266)$ & - & $30 \%(156)$ & $5.2 \%(27)$ & $16 \%(82)$ & \\
\hline \multicolumn{7}{|c|}{ Histological subtype } \\
\hline IDC, NST & $84.6 \%(436)$ & $33 \%(172)$ & $29 \%(151)$ & $06.6 \%(34)$ & $15 \%(79)$ & \multirow{5}{*}{$<0.0001$} \\
\hline ILC & $09.3 \%(48)$ & $06.6 \%(34)$ & $2 \%(10)$ & 0 & $0.77 \%(4)$ & \\
\hline Papillary & $02.3 \%(12)$ & $0.77 \%(4)$ & $1.1 \%(6)$ & $0.2 \%(1)$ & $0.2 \%(1)$ & \\
\hline Mucinous & $1.7 \%(9)$ & $1.1 \%(6)$ & $0.6 \%(3)$ & 0 & 0 & \\
\hline Others & $2 \%(10)$ & 0 & 0 & $0.2 \%(1)$ & $1.7 \%(9)$ & \\
\hline
\end{tabular}


PR-negative and HER2-positive or HER2 negative with high Ki-67 levels ), 3) HER2 enriched (ER-negative, PR-negative and HER2-positive), and (4) triple-negative (ER-negative, PR-negative, HER2-negative), the cases distribution in the cohort was $42 \%, 33 \%, 8 \%$, and $17.6 \%$ of the cases, respectively (Tab. 2).

As showed in (Fig.1), luminal B-Like breast cancers showed the highest proportion of high Ki6-7 index value, $30.3 \%$ (156) of the cohort, followed by triple-negative breast cancer, $15.9 \%$ (82), and the least was Her2 enriched subtype, 05.2\% (27). Nonetheless, luminal A-Like cancers showed the highest proportion of low Ki6-7 index value, 42\% (216), followed by lu-

Table 2. Ki-67 proliferation index and breast cancer phenotypes

\begin{tabular}{lcc}
\hline Breast cancer IHC subtype & $\begin{array}{c}\text { Low Ki-67 } \\
\%(\mathrm{n})\end{array}$ & $\begin{array}{c}\text { High Ki-67 } \\
\%(\mathrm{n})\end{array}$ \\
\hline Luminal A Like & $42(216)$ & - \\
ER+VE, PR+VE, HER2-VE & & \\
ER+VE, PR-VE, HER2-VE & & \\
Luminal B Like & $02.9(15)$ & $30.3(156)$ \\
$\quad$ ER+VE, PR+VE, HER2+VE & & \\
ER+VE, PR-VE, HER2+VE & & \\
ER+VE,PR+VE,HER2-VE & & \\
Triple-negative & & \\
ER-VE, PR -VE, HER2-VE & $01.7(9)$ & $15.9(82)$ \\
Her2 Enriched & & \\
ER-VE, PR-VE, HER2+VE & $01.9(10)$ & $05.2(27)$
\end{tabular}

minal B-Like cancers with $02.9 \%(15)$, triple-negative breast cancers were $01.7 \%(n=9)$ and HER2 enriched tumours were $1.9 \%(n=10)$. Our data discloses a statistically significant correlation between tumour grade and Ki-67 proliferative index levels among the different histopathological tumour subtypes ( $P$ values of $0<0.00001$ ).

\section{Discussion}

Breast cancer is regarded as a heterogeneous disorder, tumour detailed assessment and categorization into certain immuno-histochemical subtype based on molecular studies is recommended to predict disease prognosis and facilitate management decisions and planning. In addition to the Ki-67 proliferation index, many other biomarkers and online tools have been utilised to assess the prognosis and disease recurrence prediction. These biological criteria include ER (Oestrogen Receptor) expression, PR (Progesterone Receptor) expression, HER2 (Human Epidermal Growth Factor Receptor - type 2) expression, and Oncotype DX recurrence score, where the online tools include; NPI (Nottingham Prognostic Index) and PREDICT (Tab. 3). This is required to identify cases associated with a sufficiently high risk of disease relapse to warrant them adjuvant chemotherapy and prolonged hormonal manipulation treatment if appropriate. Assessment of the Ki-67 proliferation index is based on IHC (immune-histochemical) staining of the tumour cells for the Ki67 protein, it is detected in the active course of the cell cycle ( Late G1, S, G2, and M), and not detected during

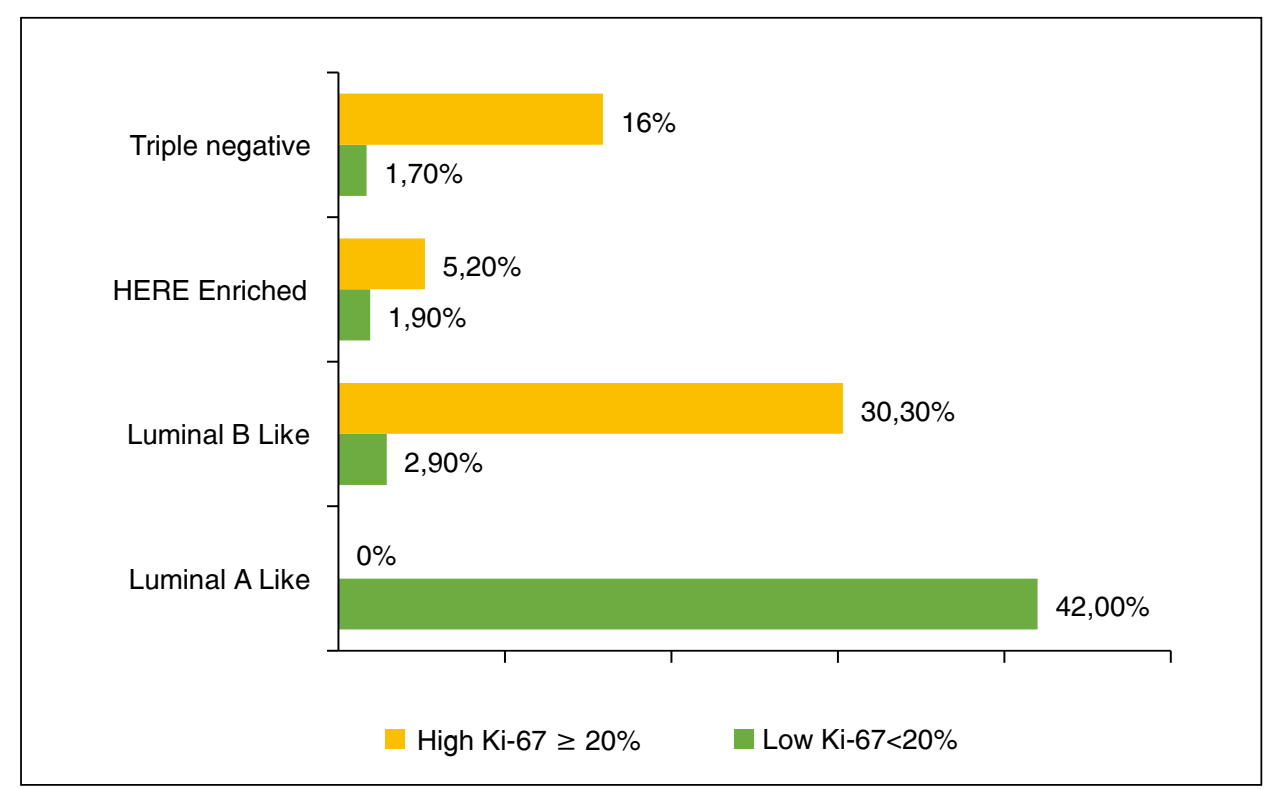

Figure 1. Ki-67 proliferation index value in different subtypes of breast cancers based on immuno-histo-chemistry classification 
G0 and early G1 [1, 10, 11]. The Ki-67 proliferative index has value in cancer diagnosis as a cell proliferation indicator, the cancer tissues show a significantly higher expression of $\mathrm{Ki}-67$ proliferative index than in

Table 3. Conventional and genomic prognostic factors as well as online prognostic tools [1]

\begin{tabular}{|c|c|}
\hline \multirow[t]{4}{*}{ Patient factors } & Age \\
\hline & Gender \\
\hline & Fitness for treatment \\
\hline & Patient compliance \\
\hline \multirow[t]{6}{*}{ Tumour factor } & Size \\
\hline & Histological subtype \\
\hline & Histological grade \\
\hline & Axillary lymphadenopathy \\
\hline & Lympho-vascular invasion \\
\hline & Extra-capsular extension \\
\hline \multirow[t]{4}{*}{$\mathrm{IHC}$} & ER \\
\hline & PR \\
\hline & HER2 \\
\hline & Ki-67 Proliferation index \\
\hline \multirow[t]{3}{*}{ Genetic mutations } & BRCA1 \\
\hline & BRCA2 \\
\hline & TP53 \\
\hline \multirow[t]{2}{*}{ Genomic tests } & Oncotype DX \\
\hline & MammaPrint \\
\hline Online tools & $\begin{array}{l}\text { NPI(Nottingham Prognostic Index) } \\
\text { PREDICT }\end{array}$ \\
\hline
\end{tabular}

healthy tissues [6]. Nagao et al., in 2011, presented a cohort of 119 patients treated for prostate cancer, the paper concluded that the Ki-67 proliferation index is an independent factor for survival rate, this included tumour grade and stage [12]. Nielsen et al., in 2013, published a result of a prospective 190 patient cohort with primary cutaneous melanoma. Also, this study concluded that Ki-67 proliferation index was a strong prognostic marker in primary cutaneous melanoma [7]. Some recent studies revealed that the administration of antibodies targeted against the Ki-67 protein was shown to result in a slower rate of cell division. This fact makes Ki-67 a promising factor for targeted molecular cancer therapy. ASOs (Antisense oligonucleotide) are small-sized single-stranded nucleic acids that are used as Ki-67 peptide nucleic acid antagonists affects the cancer cells proliferation and apoptosis [13]. Another field where the Ki-67 proliferation index could be useful is monitoring the tumour response to upfront chemotherapy prior to surgery, Mukai et al, in 2014, in research related to $237 \mathrm{HER} 2$ positive breast cancer patients. The response to upfront chemotherapy was assessed using pre-chemotherapy, mid-chemotherapy (3 cycles of paclitaxel and trastuzumab) and postoperative Ki-67 proliferation index. The cohort divided into the control arm or the Ki-67 response-guided arm (Ki-67 arm).

The control arm continued the same treatment regardless of the achieved result of the Ki-67 proliferation index, where $\mathrm{Ki}-67$ arm group further treatment was modified according to the interim Ki-67 index. They have found that there was a linear correlation between the $\mathrm{Ki}-67$ proliferation index reduction rate at interim evaluation and the pathological complete response to upfront chemotherapy [14]. In addition to its diagnostic

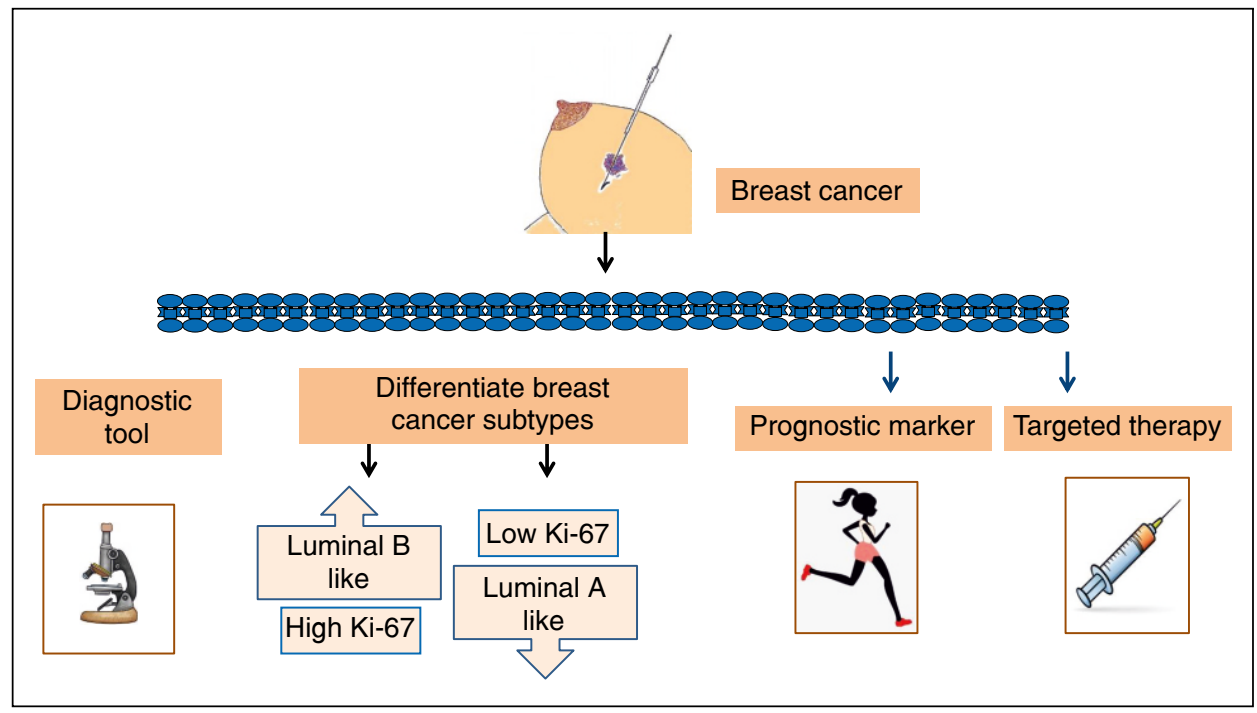

Figure 2. Current and potential future utilization of Ki-67 proliferation index in cancer management 
Table 4. Breast cancer molecular subtypes and their criteria [15, 17-19, 25]

\begin{tabular}{|c|c|c|c|c|c|}
\hline Breast cancer subtype & $\begin{array}{l}\text { Sex hormone, HER2 and cytokeratins } \\
\text { expression }\end{array}$ & Incidence & Ki-67 & Grade & Prognosis \\
\hline Luminal A & $\begin{array}{l}\text { ER+VE and/or PR+VE, HER2-VE, CK5/6-VE, } \\
\text { Keratin 8/18+VE }\end{array}$ & $71 \%$ & Low & Low & Good \\
\hline Luminal B & $\begin{array}{l}\text { ER+VE and/or PR+VE, HER2+VE, CK5/6-VE, } \\
\text { Keratin } 8 / 18\end{array}$ & $8 \%$ & & & \\
\hline Luminal B-like & $\mathrm{ER}+\mathrm{VE}$, any $\mathrm{PR}, \mathrm{HER} 2-\mathrm{VE}$ with high $\mathrm{K}$-i67 & & High & & \\
\hline Triple-negative "Basal-like" & ER-VE, PR -VE, HER2-VE, CK5/6+VE & $15 \%$ & & High & Poor \\
\hline HER2-enriched & ER-VE, PR-VE, HER2+, CK5/6-VE & $6 \%$ & & High & Poor \\
\hline Normal breast-like group & Not classified & & & & \\
\hline
\end{tabular}

and prognostic value, $\mathrm{Ki}-\mathrm{i} 7$ proliferation index is used to differentiate between Luminal A-like and Luminal B-like subtypes in ER+VE/HER2-VE breast cancer (Fig.2). The main histological breast cancer subtypes have been identified according to ER (oestrogen receptor), PR (progesterone receptor), and HER2 (Human Epidermal Growth Factor Receptor - type 2) expression (Tab. 4). Additional molecular classification is based on gene expression profile studies also is increasingly used to categorize different molecular subtypes of breast cancer. The subtype luminal type $A$ which is the most frequent identified breast cancer subtype is strong ER+VE and/or PR+VE/HER2-VE status. These tumours are known to be of a low histological grade tumour, and usually associated with a good prognosis, it forms about $71 \%$ of breast cancers, likely to benefit from hormonal blockade treatment and may benefit from chemotherapy $[15,16]$ in our cohort, luminal A-like tumours are encountered in $42 \%$ of cases. The luminal B subtype is weak/moderate $E R+V E$ and/or $\mathrm{PR}+\mathrm{VE}$ with HER2+VE (overexpression or amplification), this subtype has higher Ki-67 levels than luminal A tumours, and it is encountered in about $8 \%$ of breast cancers. These tumours are likely to benefit from neo/adjuvant chemotherapy and may benefit from hormonal manipulation therapy in addition to the HER2 targeted treatment [17]. Luminal B-like tumours are ER+VE and/or PR-VE with HER2-VE expression, however, has a high Ki-67 index value. The basal-like breast cancer (BLBC), given this name as they are characterized by high expression of genes characteristic of normal breast tissue basal epithelial cells as CK 5/6, CK14, CK15 and CK17. Most of them (but not all) are triple-negative breast cancer (i.e. has a receptor expression ER-VE, PR-VE, HER2-VE), encountered frequently in young premenopausal patients with high BMI. They are high-grade tumours, forms about $15 \%$ of all invasive ductal carcinoma of no special type, associated with an aggressive clinical course, often relapsing rapidly either loco-regional or as a distant metastatic disease and linked to a high mortality rate [18-20].The HER2-E (HER2 enriched) subtype tumors have HER2+VE/ER-
VE/PR-VE expression, these tumours are less common however some of them are characterized by high-grade histology and poor prognosis [21]. A recent systematic review and meta-analysis published by Schettini et al. from Naples, Italy in 2020, the paper stating that HER2-E tumours are associated with a higher likelihood of achieving a complete pathological response following neoadjuvant anti-HER2-based therapy [22]. Some previous reports has stated that, the triple-negative breast cancer is associated with the highest Ki-67 index values, where the HER2 positive tumours were in second place. Looking at the histological subtypes, the observations revealed that the metaplastic and medullary breast cancers showed a significantly higher Ki-67 proliferative index as compared to invasive ductal carcinoma, NST $[23,24]$. Our cohort showing that luminal B-like cancers has the highest Ki-67 index values (30\%), followed by triple negative breast cancer (16\%). Perez-Lopez et al., in 2016 , presented a series of 680 patients, using the Saint Gallen criteria, the cohort was divided in IHC subtypes. The prognosis of the groups was analysed. It was found the luminal B NO had the most unfavorable prognosis, the other criteria which were associated with this group are those of the luminal B tumours as PR negative, HER2 positive as well as high Ki-67 proliferation index expression [24]. Our results show that in addition to the luminal B-like cancers, other subtypes of breast cancer also has high $\mathrm{Ki}-67$ index values, these subtypes include triple-negative breast cancer (16\%) and HER2 enriched tumours (5.2\%). These tumours are less common than luminal A tumours, however, some of them are characterized by high-grade histology and poor prognosis. These findings are in accordance with the published researches data.

\section{Conclusion}

Generally, this data showed that the majority of the cases of low Ki-67 index expression belongs to the luminal A-like group, where the majority of cases with a high Ki-67 index expression are of non-luminal A-like 
subtype as luminal B-Like and triple-negative tumours $(\mathrm{P}<0.00001)$. The Ki-67 level is regarded as a helpful biomarker in breast cancer management, its expression is strongly associated with disease aggressiveness and prognosis, also it has an additional value, currently is considered as a promising therapeutic target in breast cancer.

\section{References}

1. Kanyılmaz G, Yavuz BB, Aktan M, et al. Prognostic importance of Ki-67 in breast cancer and its relationship with other prognostic factors. Eur J Breast Health. 2019; 15(4): 256-261, doi: 10.5152/ejbh.2019.4778, indexed in Pubmed: 31620685.

2. Al-Zawi A. Ki -67 proliferative index as a predictive tool for axillary pathological complete response in node-positive breast cancer International Journal of Medical Science. 2020; 7(11): 1-4, doi: 10.14445/23939117/ijms-v7i11p101.

3. Fulton R. Getting a Grip on Ki-67. Appl Immunohistochem Mol Morphol. 2021; 29(2): 83-85, doi: 10.1097/PAl.0000000000000908, indexed in Pubmed: 33559991.

4. Caputo A, D'Antonio A, Memoli D, et al. Ki67 in gleason pattern 3 as a marker of the presence of higher-grade prostate cancer. Appl Immunohistochem Mol Morphol. 2021; 29(2): 112-117, doi: 10.1097/PAl.0000000000000835, indexed in Pubmed: 32107350

5. Colón-Caraballo M, García M, Mendoza A, et al. Human endometriosis tissue microarray reveals site-specific expression of estrogen receptors, progesterone receptor, and Ki67. Appl Immunohistochem Mol Morphol. 2019; 27(7): 491-500, doi: 10.1097/PAl.0000000000000663, indexed in Pubmed: 29629944.

6. Hu HY, Liu Hu, Zhang JW, et al. Clinical significance of Smac and Ki67 expression in pancreatic cancer. Hepatogastroenterology. 2012 59(120): 2640-2643, doi: 10.5754/hge12071, indexed in Pubmed: 22534537

7. Nielsen PS, Riber-Hansen R, Raundahl J, et al. Automated quantification of MART1-verified Ki67 indices by digital image analysis in melanocytic lesions. Arch Pathol Lab Med. 2012; 136(6): 627-634, doi: 10.5858/arpa.2011-0360-OA, indexed in Pubmed: 22646269.

8. Melling N, Kowitz CM, Simon R, et al. High Ki67 expression is an independent good prognostic marker in colorectal cancer. J Clin Pathol. 2016; 69(3): 209-214, doi: 10.1136/jclinpath-2015-202985, indexed in Pubmed: 26281861.

9. Yagi T, Inoue N, Yanai A, et al. Prognostic significance of geminin expression levels in Ki67-high subset of estrogen receptor-positive and HER2-negative breast cancers. Breast Cancer. 2016; 23(2): 224-230, doi: 10.1007/s12282-014-0556-9, indexed in Pubmed: 25082658.

10. Pathmanathan N, Balleine RL. Ki67 and proliferation in breast cancer. J Clin Pathol. 2013; 66(6): 512-516, doi: 10.1136/jclinpath-2012-201085, indexed in Pubmed: 23436927.
11. Saad Abdalla Al-Zawi A, Syed A. Ki67 Proliferation index as a prognostic and predictive tool for pathological response after upfront chemotherapy in breast cancer. . Paripex indian Journal of Research 2020; 9(11).

12. Mukai H, Yamaguchi T, Takahashi M, et al. Ki-67 response-guided preoperative chemotherapy for HER2-positive breast cancer: results of a randomised Phase 2 study. Br J Cancer. 2020; 122(12): 1747-1753, doi: 10.1038/s41416-020-0815-9, indexed in Pubmed: 32238920.

13. Zheng JN, Sun YF, Pei DS, et al. Anti-Ki-67 peptide nucleic acid affects the proliferation and apoptosis of human renal carcinoma cells in vitro. Life Sci. 2005; 76(16): 1873-1881, doi: 10.1016/j.Ifs.2004.10.034, indexed in Pubmed: 15698864

14. Mukai H, Yamaguchi T, Takahashi M, et al. Ki-67 response-guided preoperative chemotherapy for HER2-positive breast cancer: results of a randomised Phase 2 study. Br J Cancer. 2020; 122(12): 1747-1753, doi: 10.1038/s41416-020-0815-9, indexed in Pubmed: 32238920.

15. Millikan RC, Newman B, Tse CK, et al. Epidemiology of basal-like breast cancer. Breast Cancer Res Treat. 2008; 109(1): 123-139, doi: 10.1007/s10549-007-9632-6, indexed in Pubmed: 17578664.

16. Wiechmann L, Sampson M, Stempel M, et al. Presenting features of breast cancer differ by molecular subtype. Ann Surg Oncol. 2009; 16(10): 2705-2710, doi: 10.1245/s10434-009-0606-2, indexed in Pubmed: 19593632.

17. Bustreo S, Osella-Abate S, Cassoni P, et al. Optimal Ki67 cut-off for luminal breast cancer prognostic evaluation: a large case series study with a long-term follow-up. Breast Cancer Res Treat. 2016; 157(2): 363371, doi: 10.1007/s10549-016-3817-9, indexed in Pubmed: 27155668.

18. Badowska-Kozakiewicz AM, Budzik MP. Immunohistochemical characteristics of basal-like breast cancer. Contemp Oncol (Pozn). 2016; 20(6): 436-443, doi: 10.5114/wo.2016.56938, indexed in Pubmed: 28239279

19. Toft DJ, Cryns VL. Minireview: Basal-like breast cancer: from molecula profiles to targeted therapies. Mol Endocrinol. 2011; 25(2): 199-211, doi: 10.1210/me.2010-0164, indexed in Pubmed: 20861225.

20. Iwase $\mathrm{H}$, Kurebayashi J, Tsuda $\mathrm{H}$, et al. Clinicopathological analyses of triple negative breast cancer using surveillance data from the Registration Committee of the Japanese Breast Cancer Society. Breast Cancer. 2010; 17(2): 118-124, doi: 10.1007/s12282-009-0113-0, indexed in Pubmed: 19466512.

21. Perou CM, Børresen-Dale AL. Systems biology and genomics of breast cancer. Cold Spring Harb Perspect Biol. 2011; 3(2), doi: 10.1101/cshperspect.a003293, indexed in Pubmed: 21047916.

22. Schettini F, Pascual T, Conte B, et al. HER2-enriched subtype and pathological complete response in HER2-positive breast cancer: A systematic review and meta-analysis. Cancer Treat Rev. 2020; 84: 101965, doi: 10.1016/i.ctrv.2020.101965, indexed in Pubmed: 32000054

23. Hashmi AA, Hashmi KA, Irfan M, et al. Ki67 index in intrinsic breast cancer subtypes and its association with prognostic parameters. BMC Res Notes. 2019; 12(1): 605, doi: 10.1186/s13104-019-4653-x, indexed in Pubmed: 31547858.

24. Pérez-López ME, García-Gómez J, Alves MT, et al. Ki-67 is a prognostic marker for hormone receptor positive tumors. Clin Transl Oncol. 2016; 18(10): 996-1002, doi: 10.1007/s12094-015-1472-y, indexed in Pubmed: 26742937

25. Soliman NA, Yussif SM. Ki-67 as a prognostic marker according to breast cancer molecular subtype. Cancer Biol Med. 2016; 13(4): 496-504, doi: 10.20892/j.issn.2095-3941.2016.0066, indexed in Pubmed: 28154782. 